\title{
DON: Deep Learning and Optimization-Based Framework for Detection of Novel Coronavirus Disease Using X-ray Images
}

\author{
Gaurav Dhiman $^{1} \cdot$ V. Vinoth Kumar ${ }^{2} \cdot$ Amandeep Kaur $^{3} \cdot$ Ashutosh Sharma $^{4}$
}

Received: 17 September 2020 / Revised: 11 January 2021 / Accepted: 1 February 2021 / Published online: 15 February 2021

(c) International Association of Scientists in the Interdisciplinary Areas 2021

\begin{abstract}
In the hospital, a limited number of COVID-19 test kits are available due to the spike in cases every day. For this reason, a rapid alternative diagnostic option should be introduced as an automated detection method to prevent COVID-19 spreading among individuals. This article proposes multi-objective optimization and a deep-learning methodology for the detection of infected coronavirus patients with X-rays. J48 decision tree method classifies the deep characteristics of affected X-ray corona images to detect the contaminated patients effectively. Eleven different convolutional neuronal network-based (CNN) models were developed in this study to detect infected patients with coronavirus pneumonia using X-ray images (AlexNet, VGG16, VGG19, GoogleNet, ResNet18, ResNet500, ResNet101, InceptionV3, InceptionResNetV2, DenseNet201 and XceptionNet). In addition, the parameters of the $\mathrm{CNN}$ profound learning model are described using an emperor penguin optimizer with several objectives (MOEPO). A broad review reveals that the proposed model can categorise the X-ray images at the correct rates of precision, accuracy, recall, specificity and F1-score. Extensive test results show that the proposed model outperforms competitive models with well-known efficiency metrics. The proposed model is, therefore, useful for the realtime classification of X-ray chest images of COVID-19 disease.
\end{abstract}

Keywords Coronavirus $\cdot$ COVID- $19 \cdot \mathrm{MOEPO} \cdot \mathrm{CNN} \cdot \mathrm{J} 48 \cdot$ Deep learning $\cdot$ Optimization

\section{Introduction}

The latest coronavirus (COVID-19) pandemic started in December 2019 in Wuhan, China, and has become a major global public health issue [1,2]. The pandemic virus COVID-19 was also named SARS-CoV-2 [3], a severe acute syndrome of coronavirus. Coronaviruses $(\mathrm{CoV})$ are a large group of viruses that cause residual conditions such as Middle East Respiratory Syndrome (MERS-CoV) and Extreme Acute Respiratory Syndrome (SARS-CoV). The new genus

Gaurav Dhiman

gdhiman0001@gmail.com

1 Department of Computer Science, Government Bikram College of Commerce, Punjabi University, Patiala 147001, Punjab, India

2 Department of Computer Science and Engineering, MVJ College of Engineering, Bangalore, India

3 Department of Computer Science, Sri Guru Granth Sahib World University, Fatehgarh Sahib, Punjab, India

4 Institute of Computer Technology and Information Security, Southern Federal University, Rostov-on-Don, Russia
Coronavirus (COVID-19) was detected in 2019 and never before observed in humans.

The animal-to-human contamination of the zoonotic coronaviruses was discovered by [4]. Research has shown the contamination of SARS-CoV by muscular cats inhuman beings and the contamination of MERS-CoV by dromedary in human beings [5]. Table 1 shows the definition, mortality rate, and origin of the coronavirus.

Respiratory transmission from person to person caused the rapid spread of the disease. The signs of infection include respiratory problems, fatigue, cough and dyspnea. Severe acute respiratory syndromes, septic shock, multi-organ failure and death are more severely affected by the disease [6]. Men have been found to be sicker than women and children aged 0-9 years of age have no death [7]. In cases of COVID-19 pneumonia, respiratory levels were shown to be higher than in people with health [8]. Even with the increasing demand for intensive care facilities in many developed countries, the health system has come to a standstill. The diagnosis of COVID-19 should be confirmed as a key indicator for reverse transcription polymerase or hospitalisation by gene sequencing for respiratory or blood samples, as stated 
Table 1 Detail of coronavirus

\begin{tabular}{|c|c|c|c|}
\hline $\mathrm{CoV}$ & Year & Origin & $\begin{array}{l}\text { Mortal- } \\
\text { ity rate } \\
(\%)\end{array}$ \\
\hline SARS & 2002 & $\begin{array}{l}\text { Guangdong province, } \\
\text { China }\end{array}$ & 10 \\
\hline MERS & 2013 & Saudi Arabia & 34 \\
\hline COVID-19 & 2019 & Wuhan, China & 3.4 \\
\hline
\end{tabular}

by the Chinese government. The current public health emergency causes the low sensitivity of the RT-PCR to obstruct the detection and treatment of many COVID-19 patients. Moreover, because of the extremely infectious nature of the virus, a wider population is at risk of infection [9]. The diagnoses now include all the individuals who display the common chest pneumonia pattern COVID-19 instead of the patients who wait for positive virus tests. This method allows authorities to isolate and treat patients quicker. Some patients survive from irreversible lung failure even though death does not occur at COVID-19. COVID-19 also opens holes in the lungs like the SARS according to the World Health Organisation, giving them a 'honeycomb-like appearance' [7]. One of the methods used to treat pneumonia is the computed chest tomography (CT). Automated image analysis tools for the detection, quantification and surveillance of corona virus were developed on the basis of artificial intelligence (AI) and to differentiate between patients with coronavirus and disease free [10]. In a Fei et al. research [11], a deep-learning system was developed to automatically segment all lung and infection places using chest CT. Xiaowei et al. [12] aimed at developing an early model for the diagnosis, using $\mathrm{CT}$ images and in-depth education techniques, of COVID-19 pneumonia and influenza-A viral pneumonia in a stable situation. In the Shuai et al. [13] research, they developed a deep-learning system based on the COVID-19 radiographic changes of images taken from the $\mathrm{CT}$ that can draw out the graphical characteristics of COVID-19 before pathogenic testing, thus saving crucial time for the diagnosis of the disease. Hamimi's [14] analysis of MERS-CoV found that characteristics such as pneumonia can be present in the chest X-ray and CT. Data-mining techniques were used in the Xuanyang et al. research [15] to differentiate between SARS and conventional pneumonia based on X-ray images. On 41 COVID-19 patients, Huang et al. [16] identified the clinical characteristics, which indicate that cough, heavy, myalgia, or fatigue were typical onset symptoms. Both 41 patients had pneumonia and the chest CT test was anomalous. The first proof of human COVID-19 transmission was found at the University of Hong Kong by the Kok-KH team [17]. Zhao et al. [18] proposed a mathematical model to estimate the real numbers of cases reported in COVID-19 during the first half of January 2020. They concluded that the number of cases not reported was 469 from 1 to 15 January 2020. They also announced that after 17 January 2020, cases increased 21-fold. Nishiura et al. [19] suggested a model prediction of the COVID-19 infection rate in Wuhan, China on 29-31 January 2020 based on data from 565, Japanese citizens evacuated from Wuhan. They assume that the predicted rate is $9.5 \%$ and the mortality rate is from 0.3 to $0.6 \%$. However, the number of Japanese people evacuated from Wuhan is limited and insufficient to estimate infection and death. Tang et al. [20] proposed a mathematical model to assess the probability of transmission for COVID-19. They concluded that 6.47 simple reproductions could take place. It also estimated the number of confirmed cases in 7 days (23-29 January 2020). Moreover, they expected the best possible outcome after 2 weeks (from 23 January 2020). The data for estimating sustainable human-to-human transmission of COVID-19 from 47 patients were used in [21]. The author concluded that 0.4 is transmitted, but that 0.012 is transmitted if the time for hospitalisation effects is half the time the results are tested. The authors presented a model of an assessment of risk of death for COVID-19 in [22]. The estimates for two different cases are $5.1 \%$ and $8.4 \%$. The reproductive number for both scenarios was also measured at 2.1 and 3.2. Schemes have shown that a COVID-19 $\mathrm{X}$-ray pandemic could occur to scan the body for fractures, bone dislocation, lung infections, pneumonia, and tumours. CT scanning is a kind of state-of-the-art X-ray device that explores the very soft nature and transparency of the active part of the body of soft internal tissues and organs [23]. Radiation is easier, stronger, more effective and less hazardous than CT. If COVID-19 pneumonia is not recognised and treated quickly, mortality can increase.

We have presented an automated COVID-19 prevision based on a deep convolution network using a pre-trained transmission model and chest X-ray image. The chest X-ray of 50 COVID-19 patients are taken from Dr. Joseph Cohen's open-source GitHub [24] repository. This dataset is used as a deep extractor of features based on a profound learning collection such as AlexNet, VGG16, VGG19, GoogleNet, ResNet18, ResNet50 and ResNet101. These deep-sensed models have the deep features categorised according to the $\mathrm{J} 48$ algorithm. The parameter tuning is influenced by deep-learning models. To eliminate this problem, a multiobjective algorithm [25] is used to efficiently modify CNN model parameters. Finally, we verify the findings using deep extraction methods (see Fig. 1).

The rest of this paper is structured as follows: Sect. 2 presents the optimization process used in this research. In Sects. 3 and 4, deep learning and J48 models are discussed. The dataset description is given in Sect. 5 followed by the performance metrics in Sect. 6. Experimental results and discussions are discussed in Sect. 7. Finally, conclusions are presented in Sect. 8. 
Fig. 1 COVID-19 classification approach

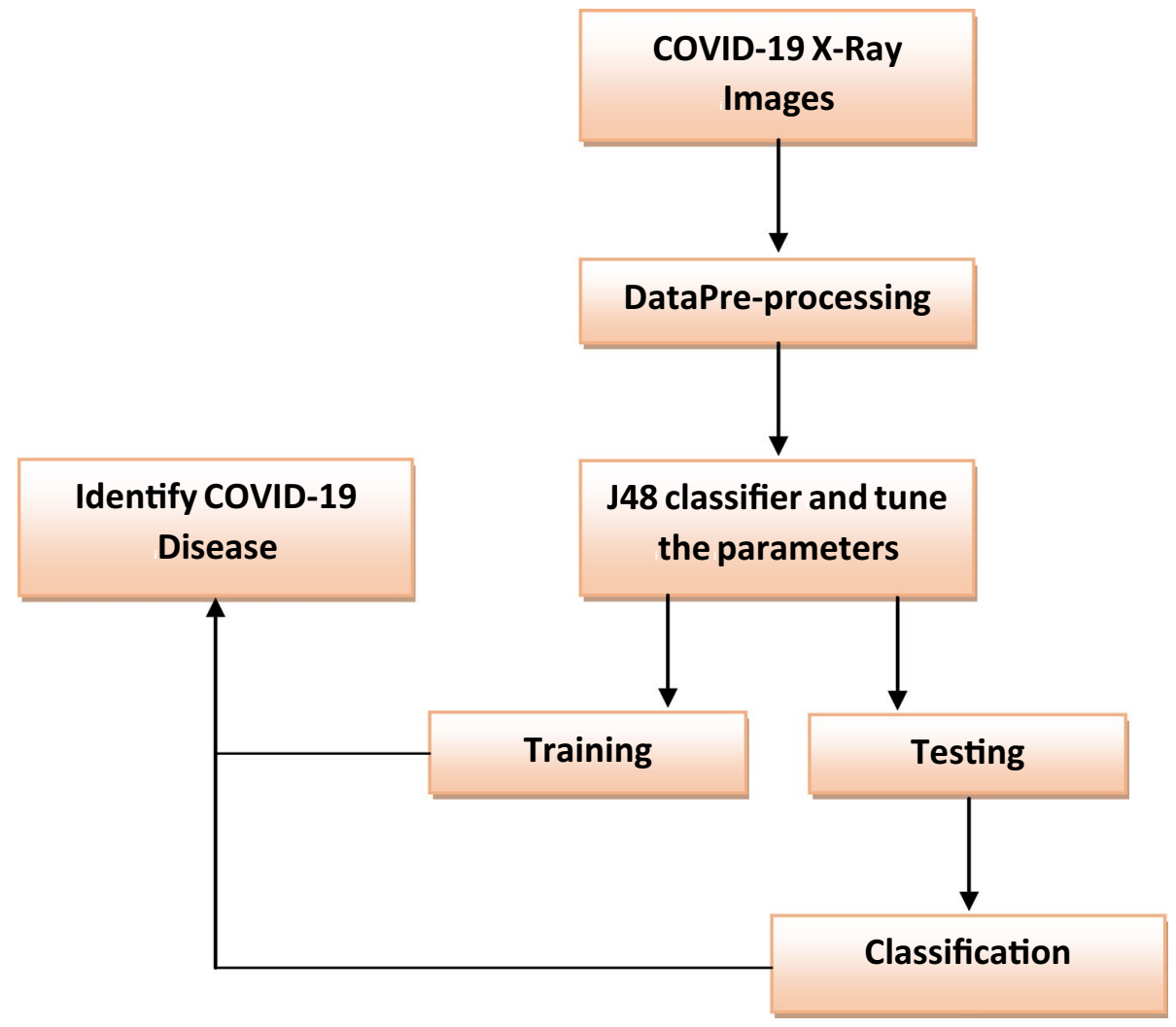

\section{Optimization}

\subsection{Emperor Penguin Optimizer (EPO)}

Optimization is a process to find the optimal number of solutions [26-34]. The emperor penguin optimizer [35] is encouraged from the huddling attitude of the emperor penguins which were originated in the Antarctic. Emperor penguins usually process in colonies for foraging. This huddling behaviour is the unique characteristic as observed in these social animals during foraging. Hence, in the mathematical model, the prime objective will be to identify an effective mover from the swarm. To achieve this, the distances between Emperor Penguins (EPs) $\left(Z_{\text {ep }}\right)$ are calculated followed by their temperature profile $\left(T_{\mathrm{mp}}\right)$. From this, the effective mover is identified, and the locations of other EPs are updated to get the optimum value.

The temperature profile of the Emperor Penguins is calculated as:

$T_{\mathrm{mp}}^{\prime}=\left(T_{\mathrm{mp}}-\frac{\mathrm{Itr}_{\mathrm{max}}}{k-\mathrm{Itr}_{\max }}\right)$,

$T_{\mathrm{mp}}=\left\{\begin{array}{l}0 \text { if } R_{\mathrm{nd}}>0.5 \\ 1 \text { if } R_{\mathrm{nd}}<0.5\end{array}\right.$, where $k$ represents the recent iteration, $\mathrm{Itr}_{\max }$ presents the maximum iteration and $R_{\mathrm{nd}}$ shows the random number between $[0,1]$.

The generated huddle boundary signifies the distance of EPs to the best optimal solution. The optimum solution is determined by considering the fitness value nearer to optimal solution. The other emperor penguins updated their position depending upon the optimum solutions as:

$\overrightarrow{D_{\mathrm{eps}}}=\left|X s(\vec{A}) \cdot \overrightarrow{Z_{\mathrm{b}}(k)}-\vec{C} \cdot \overrightarrow{Z_{\mathrm{ep}}(k)}\right|$

Here, $\overrightarrow{D_{\text {eps }}}$ shows the distance from EPs to the best solution. $\vec{A}$ and $C$ are two vectors responsible for escaping from collision from other EPs. $\overrightarrow{Z_{\mathrm{b}}}$ represents the optimum solutions, $\overrightarrow{Z_{\text {ep }}}$ shows the EP's position vector. $X s()$ indicates the social forces of EPs.

Since EPs generally huddle together to maintain temperature. Thus, special care is to be taken to make them safe from collisions among the neighbours. For this reason, two vectors $(\vec{A})$ and $(\vec{C})$ are calculated as:

$\vec{A}=\left\{M \times\left(T_{\mathrm{mp}}^{\prime}+X_{\text {grid }}(\right.\right.$ acrcy $\left.\left.)\right) \times \operatorname{Rand}()\right\}-T_{\mathrm{mp}}^{\prime}$.

$\vec{C}=\operatorname{Rand}()$. 


$$
X_{\text {grid }}(\text { acrcy })=\left|\vec{Z}_{\mathrm{b}}-\vec{Z}_{\mathrm{ep}}\right| \text {. }
$$

Here, $M$ indicates the movement parameter and is set as 2 , Rand is the random value in the range $[0,1], X_{\text {grid }}$ (acrcy) shows the absolute difference between EPs and the optimal solution.

$X_{\mathrm{s}}(\vec{A})=\left(\sqrt{f \cdot e^{-\frac{k}{v}}-e^{-k}}\right)$.

Here, $e$ represents the expression function, $f$ and $v$ represent the control parameters for a better exploration and exploitation lie within the range $[2,3]$ and $[1.5,2]$, respectively. The positions of the EPs are updated as per the optimal agent obtained as:

$\vec{Z}_{\text {eps }}(k+1)=\vec{Z}_{\mathrm{b}}(k)-\vec{A} \cdot \vec{D}_{\text {eps }}$.

In Multi-objective Emperor Penguin Optimizer (MOEPO) algorithm [25], archive and grid mechanisms are used. Further to update the search agents, group selection method is employed for better exploration and exploitation. This algorithm is used to tune the parameters of CNN model.

\section{Deep-Learning Method}

Deep learning is a sub-branch of machine learning, inspired by the structure of the brain. Deep-learning approaches used in the analysis of medical images have shown positive results in many fields in recent years. Data-processing image and signal are collected using medical imagery technologies such as MRI, computer tomography (CT), and X-rays using deep-learning models. Through this study, diseases such as diabetes mellitus, brain tumours, skin cancer and breast cancer have been detected and diagnosed more easily $[36,37]$.

Convolutional neural networks (see Figs. 2, 3) are inspired by the system of the human neuron similar to traditional neural networks. Every strange number layer has a convolution layer and a pooling and subsampling layer each even number layer except for both the input and the output layer. There are 8 levels in the CNN's architecture. For each convolution, we have used 12, 8 and 6 attributes, and each is connected to five kernel pool layers. The lots were set to 100 and the cap for the sample was set to 100 times 1 .

\section{Decision Tree (J48 Algorithm)}

Decision trees are classification or regression algorithms supervised. J48 is selected by prominence and accuracy in results from the decision tree algorithms for COVID-19

Fig. 2 Neural network

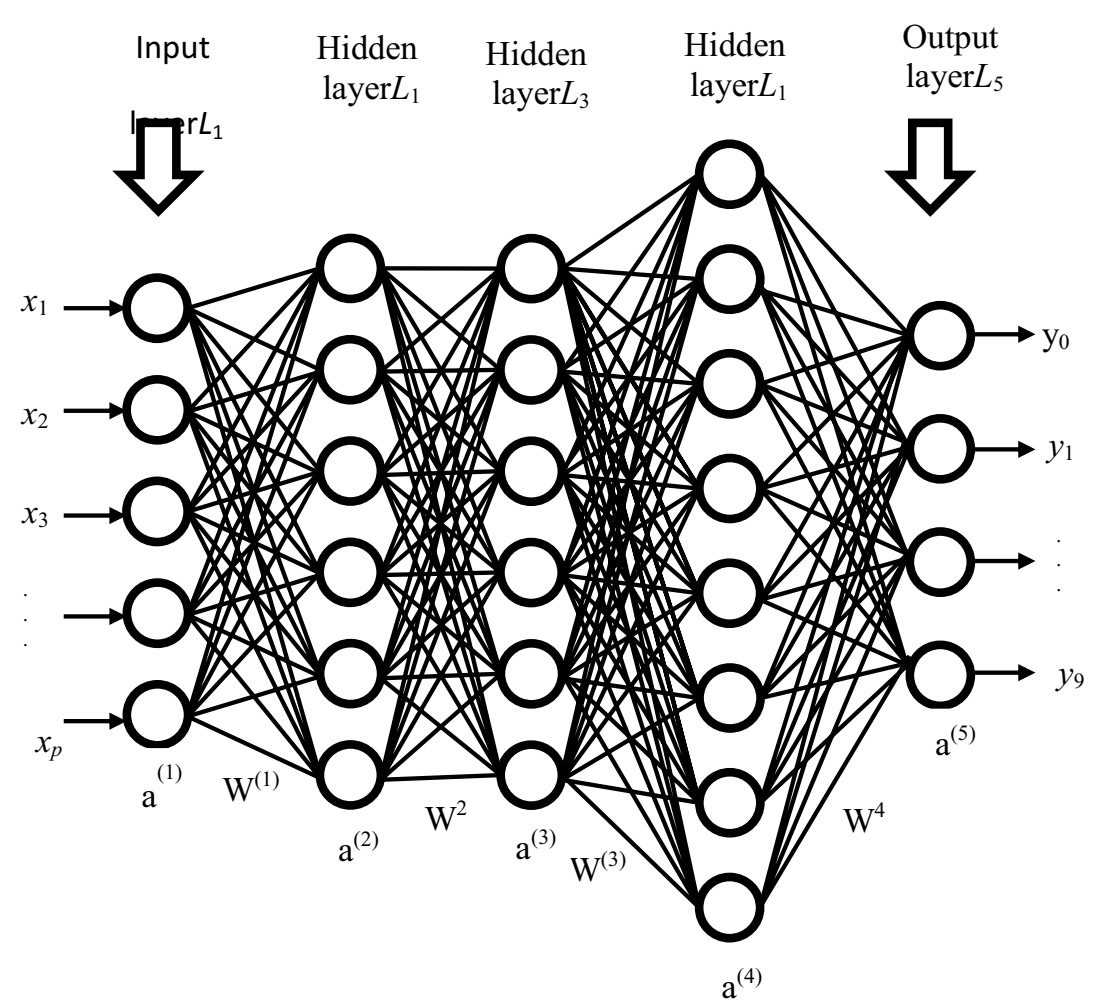




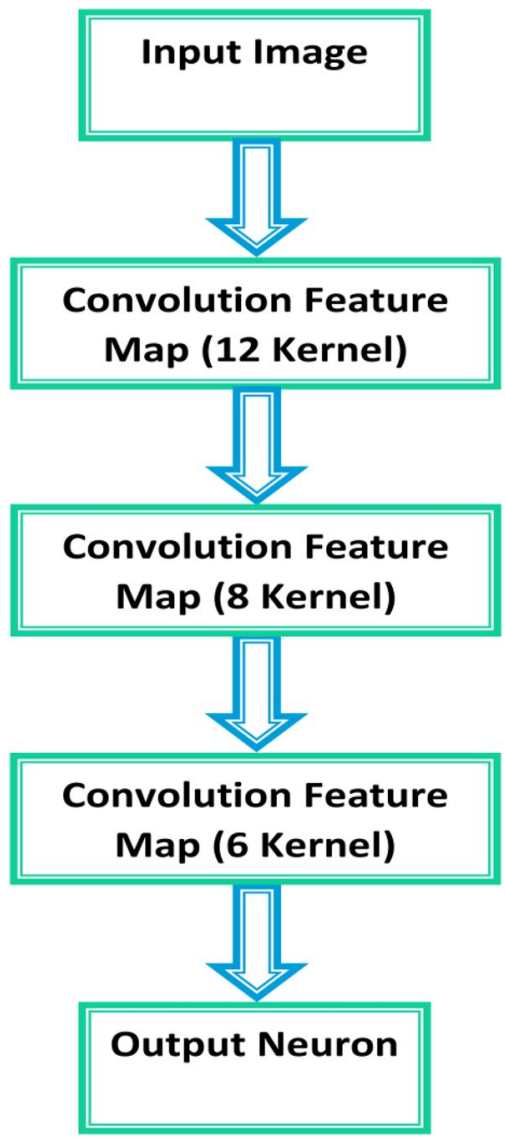

Fig. 3 Architecture of $\mathrm{CNN}$

recognition. This is an extension of ID3 (Iterative Dichotomiser 3). Additional features of J48 include (a) derivation of rules, (b) pruning of trees, (c) incompleted accounts and (d) continuous attributes. The continuous and categorical analysis of data constitutes one of the best grading algorithms. The main aim is to divide the data into a uniform class, to predict as much as possible of the variables. J48 allows classification based on either the rules defined or a decision-tab. It aims to reduce impurity or data volatility. The list of attributes for the control of continuous attributes is divided by a threshold and separated by those below and below or equal to threshold. However, it was called "?" for missing values. These missing values were not included in entropy and gain calculation.

The algorithm consists of collecting (classified) training data. A decision tree is thus generated as an output where every leaf node is a decision and a test a non-leaf node. When the root node has been tested for leaf node testing paths, a leaf node will be shown whether the variable belongs or not. After the tree is built, the tree is used to classify the tuple data in any database tuple. J48 does not know the missing values when a tree is formed. The values of this object can be determined by the established attribute value in other documents. This creates a top-down forest in the classification model. It uses uniform criteria of data division. The knowledge gain of each attribute is measured according to entropy. The most standardised characteristic is chosen for decision making. Then, a root is selected for the best attribute of the next recurrently constructed sub-trees.

\section{Dataset Description}

During this analysis, a chest X-ray image of 50 COVID-19 patients [24] was taken from the open-source GitHub repository supplied by Dr. Joseph Cohen. This archive consists of $\mathrm{X}$-ray/CT images in the chest mainly of patients with ARDS, COVID-19, Middle East Air Syndrome (MERS), pneumonia, serious acute respiratory syndrome (SARS). In addition, 50 standard chest radiation images have been used in the Kaggle repository called "Pneumonia" [38]. Our studies were performed using a dataset of 50 regular patients [38] and 50 COVID-19 patients [24]. All images were restored to a scale of $280 \times 280$ pixels in this data collection. Chest X-ray images of COVID-19 and normal patients are shown in Figs. 4 and 5.

In this study, we have established the classification COVID19 X-ray chest image classified by AlexNet, VGG16, VGG19, Google Net, ResNet18, ResNet50, ResNet101, InceptionV3, InceptionResNetV2, DenseNet201, and XceptionNet deep convolutional neural network (CNN). The deep characteristics are extracted from the fully linked layer and feed for training purposes to the classifier. The J48 decision tree classification is used in this article for the deep functions obtained from each CNN network. The classification is then performed and the performance of all classification models is measured. A particular layer eliminates the deep characteristics and functionality of CNN models. The J48 classifier for the detection of COVID-19 diseases has the characteristics. Table 2 describes the characteristic layer and vector.

\section{Performance Metrics}

The five well-known performance metrics are employed in this paper for the performance of deep-learning models.

$$
\begin{aligned}
& \text { Accuracy }=(\mathrm{TN}+\mathrm{TP}) /(\mathrm{TN}+\mathrm{TP}+\mathrm{FN}+\mathrm{FP}), \\
& \text { Recall }=\mathrm{TP} /(\mathrm{TP}+\mathrm{FN}), \\
& \text { Specificity }=\mathrm{TN} /(\mathrm{TN}+\mathrm{FP}), \\
& \text { Precision }=\mathrm{TP} /(\mathrm{TP}+\mathrm{FP}),
\end{aligned}
$$$$
\text { F1 }- \text { score }=2 \times((\text { precision } \times \text { recall }) /(\text { precision }+ \text { recall })) \text {, }
$$ 


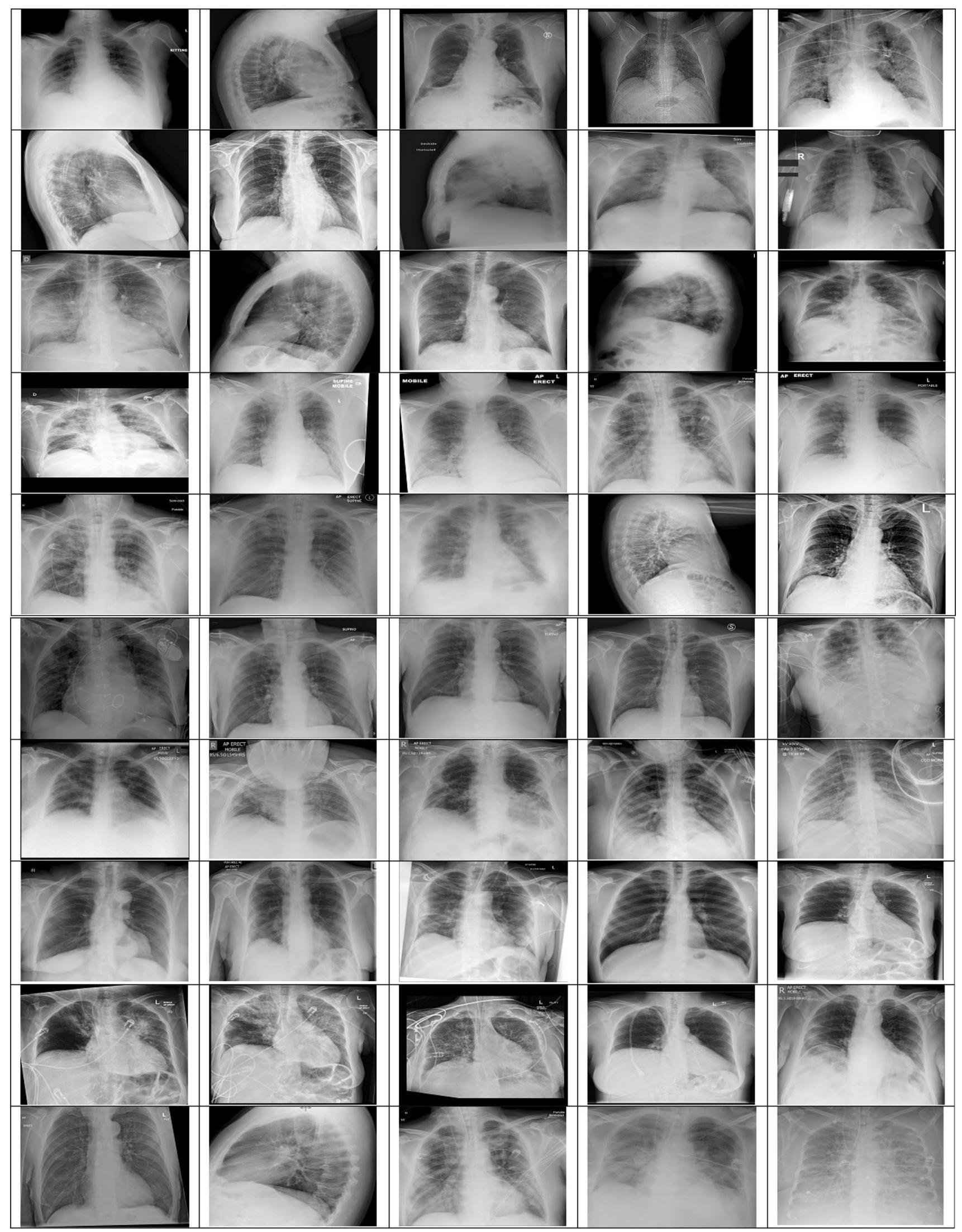

Fig. 4 X-ray images of coronavirus (COVID-19) disease effected patients 


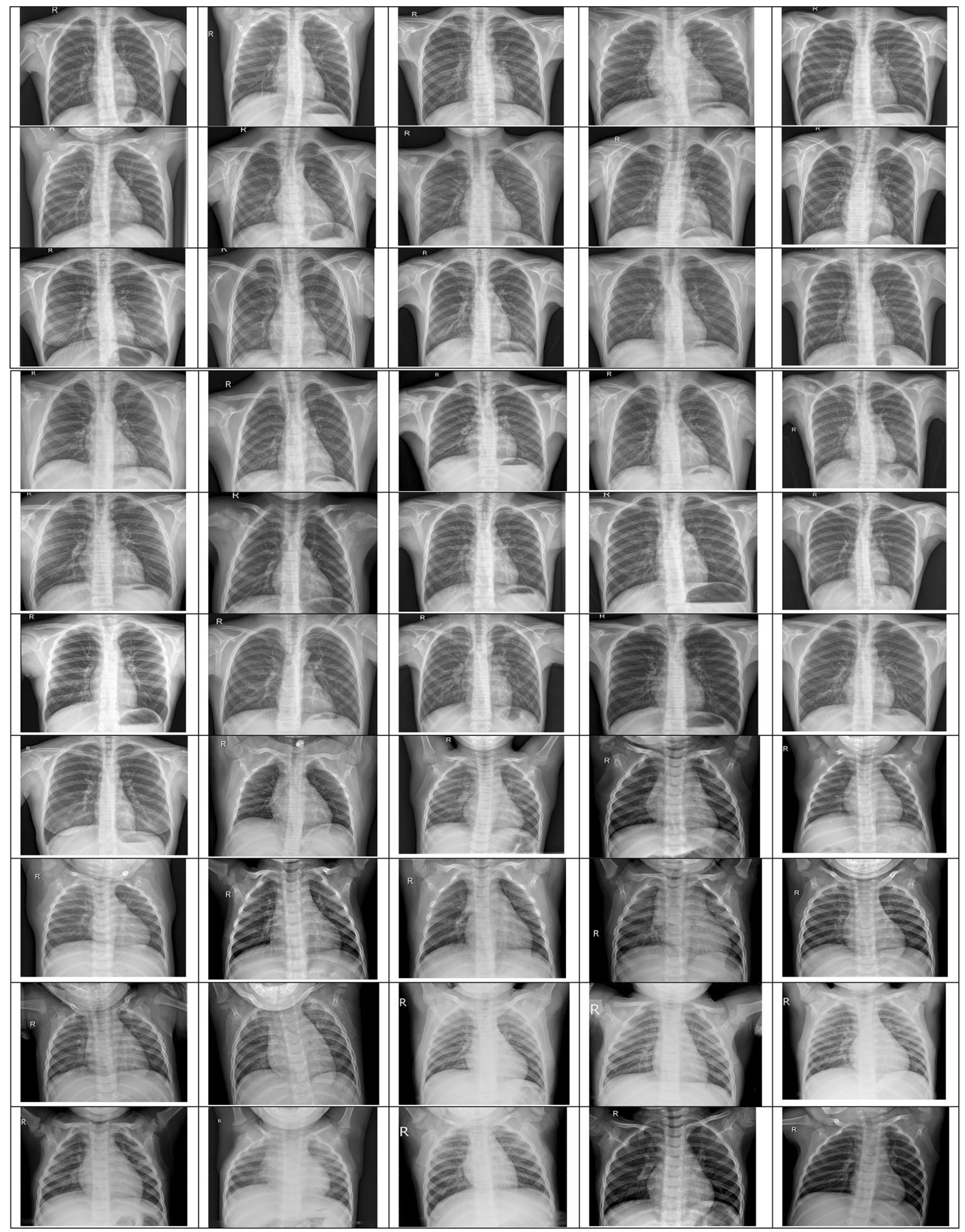

Fig. 5 X-ray images of normal patients 
Table 2 Feature layer and feature vector characteristics of CNN models

\begin{tabular}{lll}
\hline CNN models & Feature layer & Feature vector \\
\hline AlexNet & fc6 & 4096 \\
VGG16 & fc6 & 4096 \\
VGG19 & fc6 & 4096 \\
Xception & Predictions & 1000 \\
ResNet18 & Fc1000 & 1000 \\
ResNet50 & Fc1000 & 1000 \\
ResNet101 & Fc1000 & 1000 \\
InceptionV3 & Predictions & 1000 \\
InceptionResNetV2 & Predictions & 1000 \\
GoogleNet & Loss3-classifier & 1000 \\
DenseNet201 & Fc1000 & 1000 \\
\hline
\end{tabular}

where TP, FP, TN, and FN represent the number of true positive, false positive, true negative and false negative, respectively. TP is the percentage of positive (COVID-19), correctly marked by model as COVID-19; FP is the percentage of negative (normal) mislabelling (COVID-19); TN is a percentage of negative (normal) properly labelling of positive (COVID-19) that is mislabelled as negative (normal) by model; FN is the percentage of positive (COVID-19) that is wrongly labelled.

\section{Experimental Results and Discussions}

In this study, we analysed the efficiency of COVID-19 recognition classification models on the basis of $11 \mathrm{CNN}$ models. The research studies are conducted with software of MATLAB 2019a edition. All programmes are run on a Microsoft Windows environment Core i7 8th Generation and 8 GB main memory. The well-known performance metrics are used for each classifier such as Accuracy, Recall, Specificity,
Precision, and F1-Score. Table 3 results are based on an average of 50 independent simulations. The preparation, validation, and test ratio for each execution is 60:20:20 and the random selection are updated for training, validation and testing.

Figures 6, 7, 8, 9 and 10 show the performance metrics values of different models. It is observed from results that the accuracy of ResNet101 plus J48 is superior to other classification models in terms of Accuracy, Recall, Specificity, Precision, and F1-Score performance metrics. Hence, ResNet101- and J48-based CNN method result better classification for detection of COVID-19 with Accuracy, Recall, Specificity, Precision, and F1-Score are $98.50 \%, 100 \%$, $97.20 \%, 100 \%$, and $98.40 \%$, respectively.

CNN deep-learning model is efficiently detecting the COVID-19 disease from the X-ray chest images of normal and coronavirus effected patients. The detection parts from the normal images and COVID-19 images are shown in Figs. 11 and 12. It can be seen from these figures that the segmented part of the COVID-19 patients X-ray images of chest is smaller than the normal patient X-ray images. Moreover, the computational times of different classification

\section{ACCURACY}

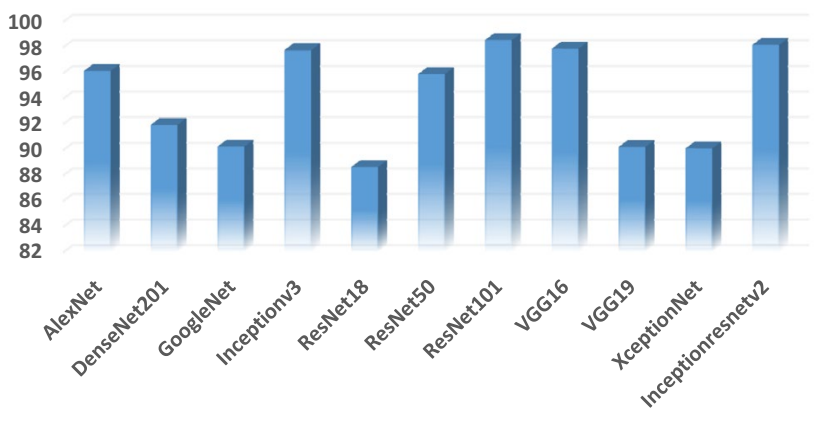

Fig. 6 The accuracy results using different classification models
Table 3 The obtained results on different models using performance metrics

\begin{tabular}{llllll}
\hline Models & Accuracy $(\%)$ & Recall (\%) & Specificity (\%) & Precision (\%) & F1-score (\%) \\
\hline AlexNet & 96.15 & 95.24 & 91.04 & 96.54 & 91.57 \\
DenseNet201 & 91.92 & 94.28 & 95.56 & 99.21 & 87.44 \\
GoogleNet & 90.25 & 94.74 & 92.47 & 96.48 & 96.21 \\
InceptionV3 & 97.76 & 90.61 & 94.68 & 90.64 & 95.47 \\
ResNet18 & 88.64 & 90.49 & 96.11 & 97.27 & 95.43 \\
ResNet50 & 95.91 & 89.55 & 93.09 & 99.64 & 92.05 \\
ResNet101 & $\mathbf{9 8 . 5 0}$ & $\mathbf{1 0 0}$ & $\mathbf{9 7 . 2 0}$ & $\mathbf{1 0 0}$ & $\mathbf{9 8 . 4 0}$ \\
VGG16 & 97.87 & 90.41 & 97.14 & 96.16 & 97.21 \\
VGG19 & 90.22 & 89.99 & 90.37 & 97.94 & 92.40 \\
XceptionNet & 90.10 & 95.47 & 92.61 & 90.54 & 90.55 \\
InceptionResNetV2 & 98.17 & 92.58 & 96.94 & 95.11 & 93.49 \\
\hline
\end{tabular}

The best results are in bold 


\section{RECALL}

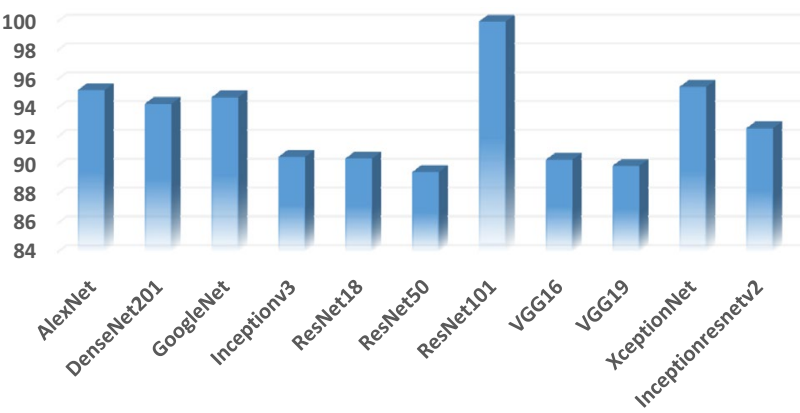

Fig. 7 The recall results using different classification models

\section{SPECIFICITY}

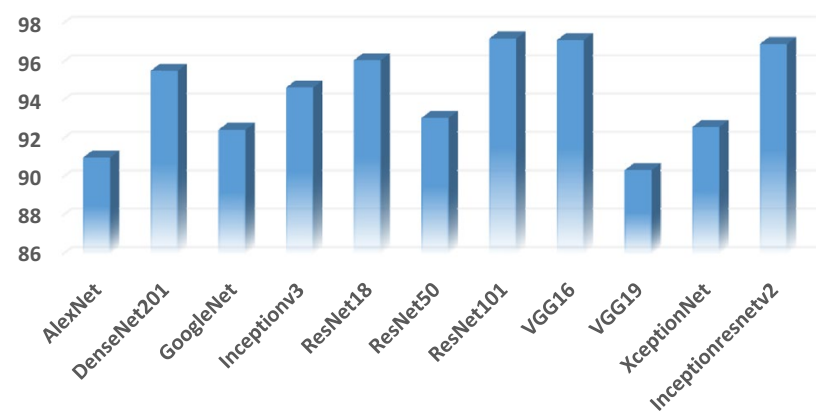

Fig. 8 The specificity results using different classification models

\section{PRECISION}

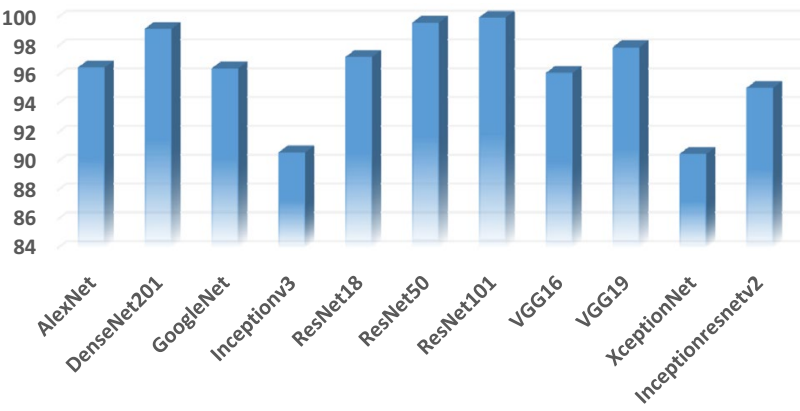

F1-SCORE

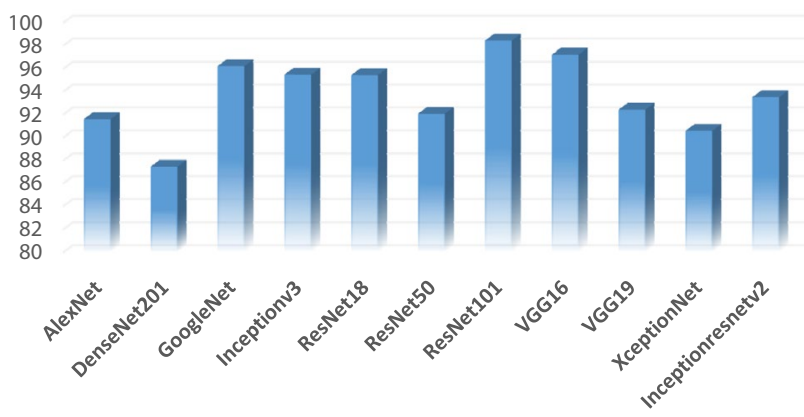

Fig. 10 The F1-score results using different classification models

models are also calculated (see Fig. 13). Overall, it can be concluded that the CNN deep-learning model and J48 decision approach is able to classify the chest X-ray images of coronavirus patients. Coronavirus identification (COVID19) is now a vital task for the physicians and researchers. The spread of COVID-19 is declared by WHO since March, 2020 as a global outbreak of the pandemic. To reduce COVID-19 spread and initiate early medical treatment for the infected individuals, it is a crucial priority to become aware of the infected individuals so that preventive procedures can be performed.

\section{Conclusions}

The contents of this paper are based on data available from the WHO, the EDC an agency of the European Union, and other official websites. The chest X-ray images used for simulation purposes are collected from the GitHub and Kaggle repositories, for coronavirus identification using deep features and J48 approach. The extraction is done using 11 pretrained CNN models and individually supplied them for J48 classification and MOEPO algorithm. Statistical research is conducted to select the best classification pattern. ResNet101 plus J48 classification model statistical performance is better than the other ten competitor models.

Fig. 9 The precision results using different classification models 


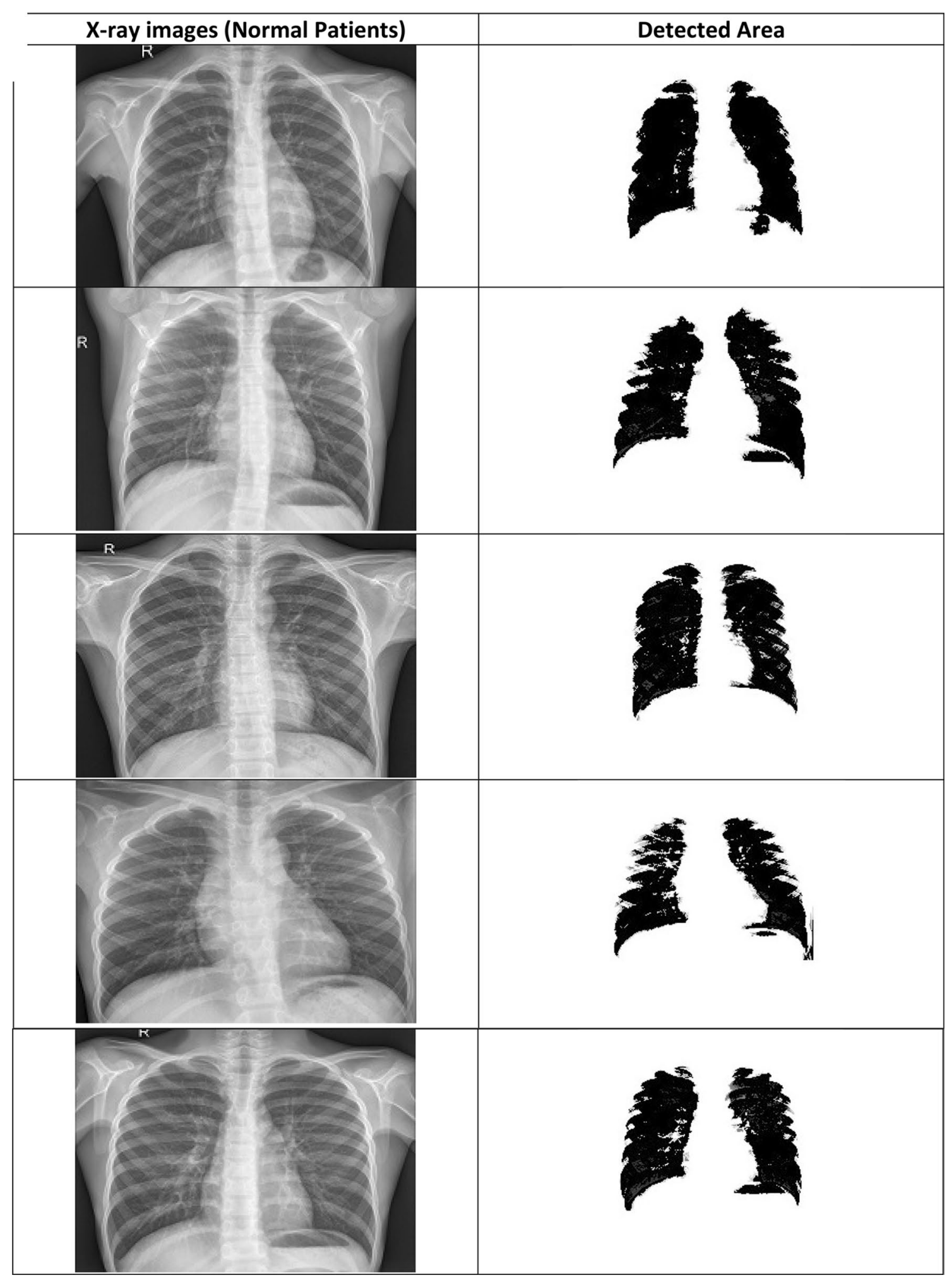

Fig. 11 Segmented chest area of normal patients using CNN approach 


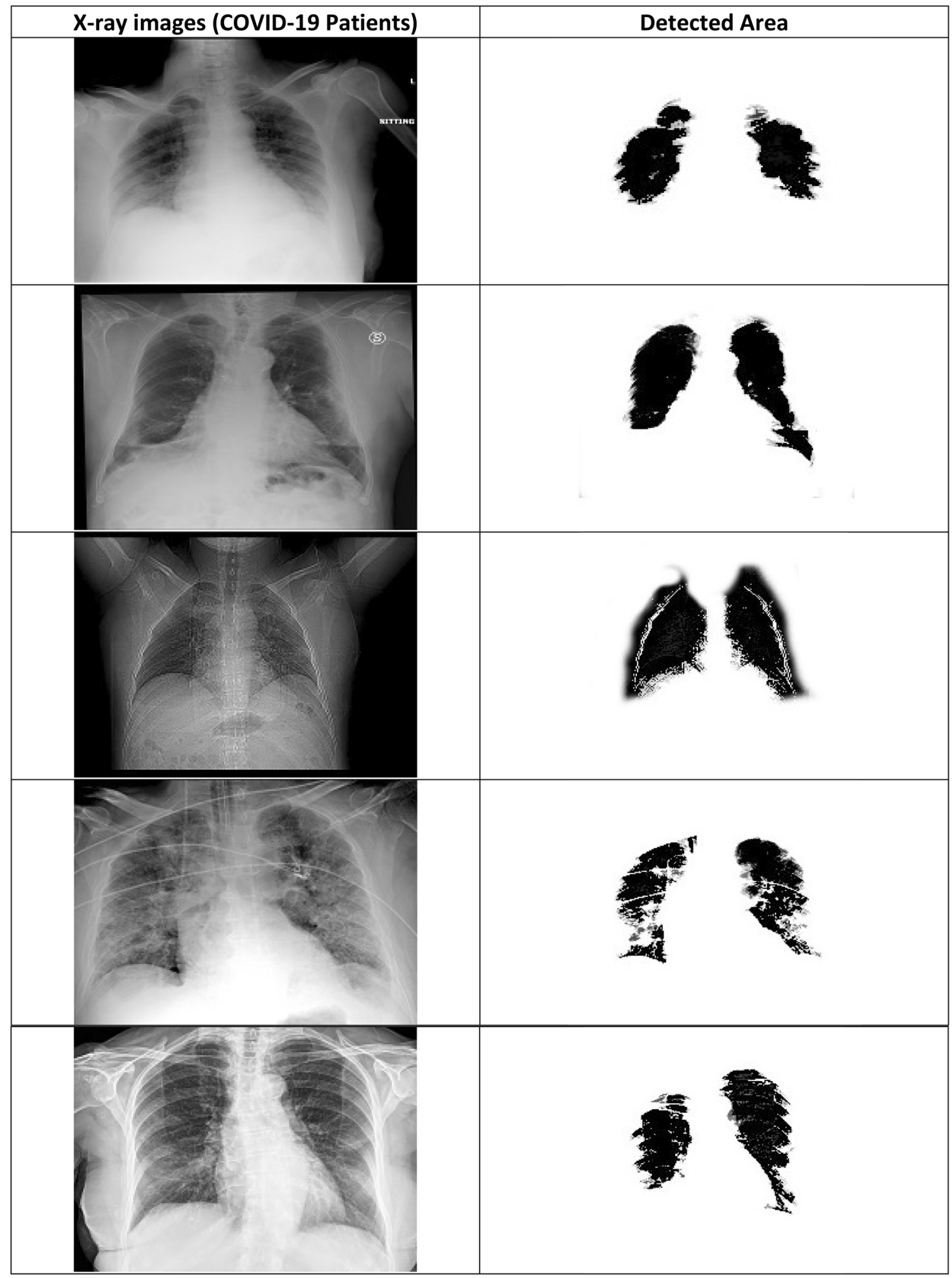

Fig. 12 Calculated computational time to predict the COVID-19 disease using different CNN models 


\section{Computational Time (in sec)}

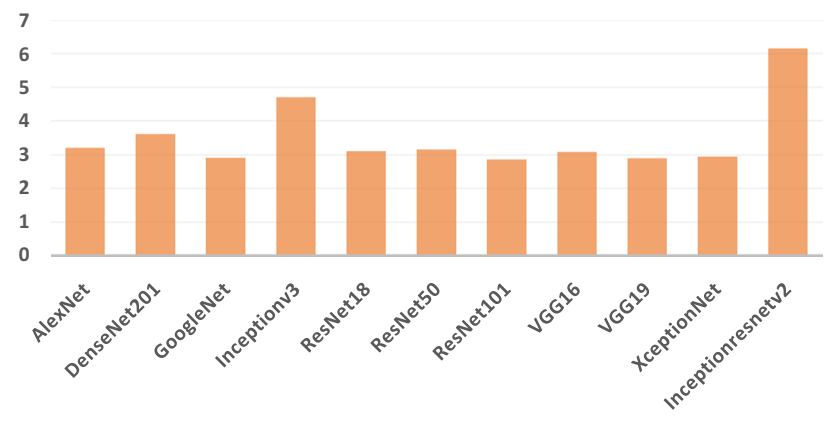

Fig. 13 Segmented chest area of COVID-19 patients using CNN approach

\section{Compliance with Ethical Standards}

Conflict of Interest The author declares that he has no conflict of interest.

Ethical Approval This research work does not involve chemicals, procedures, or equipment that have any unusual hazards inherent in their use.

Informed Consent Not required.

\section{References}

1. Roosa K, Lee Y, Luo R, Kirpich A, Rothenberg R, Hyman JM, Yan P, Chowell G (2020) Real-time forecasts of the COVID-19 epidemic in China from February 5th to February 24th, 2020. Infect Dis Model 5:256-263

2. Yan L, Zhang HT, Xiao Y, Wang M, Guo Y, Sun C, Tang X, Jing L, Li S, Zhang M, Xiao Y, Cao H, Chen Y, Ren T, Jin J, Wang F, Xiao Y, Huang S, Tan X, Huang N, Jiao B, Zhang Y, Luo A, Cao $\mathrm{Z}, \mathrm{Xu}$ H, Yuan Y (2020) Prediction of criticality in patients with severe Covid-19 infection using three clinical features: a machine learning-based prognostic model with clinical data in Wuhan. MedRxiv 143:1-18. https://doi.org/10.1101/2020.02.27.20028027

3. Stoecklin SB, Rolland P, Silue Y, Mailles A, Campese C, Simondon A, Mechain M, Meurice L, Nguyen M, Bassi C, Yamani E, Behillil S, Ismael S, Nguyen D, Malvy D, Lescure FX, Georges S, Lazarus C, Tabaï A, Stempfelet M, Enouf V, Coignard B, LevyBruhl D, Team I (2020) First cases of coronavirus disease 2019 (COVID-19) in France: surveillance, investigations and control measures, January 2020. Eurosurveillance 25(6):2000094

4. https://www.who.int/health-topics/coronavirus. 20.03.2020

5. Huang C, Wang Y, Li X, Ren L, Zhao J, Hu Y, Zhang L, Fan G, Xu J, Gu X, Cheng Z, Yu T, Xia J, Wei Y, Wu W, Xie X, Yin W, Li H, Liu M, Xiao Y, Gao H, Guo L, Xie J, Wang G, Jiang R, Gao Z, Jin Q, Wang J, Cao B (2020) Clinical features of patients infected with 2019 novel coronavirus in Wuhan, China. The Lancet 395(10223):497-506

6. Mahase E (2020) Coronavirus: covid-19 has killed more people than SARS and MERS combined, despite lower case fatality rate. The BMJ 368:m641. https://doi.org/10.1136/bmj.m641

7. https://www.nationalgeographic.com/science/2020/02/here-iswhat-coronavirus-does-to-the-body/. 20.03.2020

8. Wang Y, Hu M, Li Q, Zhang XP, Zhai G, Yao N (2020) Abnormal respiratory patterns classifier may contribute to large-scale screening of people infected with COVID-19 in an accurate and unobtrusive manner. arXiv preprint arXiv 2002(05534):1-6

9. Ai T, Yang Z, Hou H, Zhan C, Chen C, Lv W, Tao X, Sun Z, Xia L (2020) Correlation of chest CT and RT-PCR testing in coronavirus disease 2019 (COVID-19) in China: a report of 1014 cases. Radiology 200642:1-23. https://doi.org/10.1148/radiol.2020200642

10. Gozes O, Frid-Adar M, Greenspan H, Browning PD, Zhang H, Ji W, Bernheim A, Siegel E (2020) Rapid AI development cycle for the coronavirus (COVID-19) pandemic: initial results for automated detection \& patient monitoring using deep learning CT image analysis. arXiv preprint arXiv:2003.05037, 1-19

11. Shan, F., Gao, Y., Wang, J., Shi, W., Shi, N., Han, M., Xue, Z., and Shi, Y. Lung Infection Quantification of COVID-19 in CT Images with Deep Learning. arXiv preprint arXiv:2003.04655, 1-19

12. Xu X, Jiang X, Ma C, Du P, Li X, Lv S, Yu L, Chen Y, Su J, Lang G, Li Y, Zhao H, Xu K, Ruan L, Wu W (2020) Deep learning system to screen coronavirus disease 2019 pneumonia. arXiv preprint arXiv:2002.09334, 1-29

13. Wang S, Kang B, Ma J, Zeng X, Xiao M, Guo J, Cai M, Yang J, Li Y, Meng X, Xu B (2020) A deep learning algorithm using CT images to screen for corona virus disease (COVID-19). MedRxiv Preprint 57:1451. https://doi.org/10.1101/2020.02.14.20023028

14. Hamimi A (2016) MERS-CoV: Middle East respiratory syndrome corona virus: can radiology be of help? Initial single center experience. Egypt J Radiol Nuclear Med 47(1):95-106

15. Xie X, Li X, Wan S, Gong Y (2006) Mining X-ray images of SARS patients. In: Graham WJ, Simeon SJ (eds) Data mining: theory, methodology, techniques, and applications. Springer, Berlin, pp 282-294 (ISBN: 3540325476)

16. Huang C, Wang Y, Li X et al (2020) Clinical features of patients infected with 2019 novel coronavirus in Wuhan, China. Lancet. https://doi.org/10.1016/S0140-6736(20)30183-5

17. Chan JF, Yuan S, Kok KH et al (2020) A familial cluster of pneumonia associated with the 2019 novel coronavirus indicating person-to-person transmission: a study of a family cluster. Lancet. https://doi.org/10.1016/S0140-6736(20)30154-9

18. Zhao S, Musa SS, Lin Q, Ran J, Yang G, Wang W, Lou Y, Yang L, Gao D, He D et al (2020) Estimating the unreported number of novel coronavirus (2019-nCoV) cases in China in the first half of january 2020: a data-driven modelling analysis of the early outbreak. J Clin Med 9:388

19. Nishiura H, Kobayashi T, Yang Y, Hayashi K, Miyama T, Kinoshita R, Linton NM, Jung SM, Yuan B, Suzuki A et al (2020) The rate of underascertainment of novel coronavirus (2019-nCoV) infection: estimation using Japanese passengers data on evacuation flights. J Clin Med 9:419

20. Tang B, Wang X, Li Q, Bragazzi NL, Tang S, Xiao Y, Wu J (2020) Estimation of the transmission risk of the 2019-nCoV and its implication for public health interventions. J Clin Med 9:462

21. Thompson RN (2020) Novel coronavirus outbreak in Wuhan, China, 2020: intense surveillance is vital for preventing sustained transmission in new locations. J Clin Med 9:498

22. Jung SM, Akhmetzhanov AR, Hayashi K, Linton NM, Yang Y, Yuan B, Kobayashi T, Kinoshita R, Nishiura H (2020) Real time estimation of the risk of death from novel coronavirus (2019$\mathrm{nCoV}$ ) infection: Inference using exported cases. J Clin Med 9:523

23. https://biodifferences.com/difference-between-X-ray-and-ctscan.html

24. Open database of COVID-19 cases with chest X-ray or CT images https://github.com/ieee8023/covid-chestxray-dataset

25. Harsimran K, Rai A, Bhatia SS, Dhiman G (2020) MOEPO: a novel multi-objective emperor penguin optimizer for global optimization: special application in ranking of cloud service providers. Eng Appl Artif Intell 96:104008 
26. Gaurav H, Kumar V (2017) Spotted hyena optimizer: a novel bio-inspired based metaheuristic technique for engineering applications. Adv Eng Softw 114:48-70

27. Dhiman G, Kumar V (2018) Multi-objective spotted hyena optimizer: a multi-objective optimization algorithm for engineering problems. Knowl-Based Syst 150:175-197

28. Dhiman G, Kumar V (2019) Seagull optimization algorithm: theory and its applications for large-scale industrial engineering problems. Knowl-Based Syst 165:169-196

29. Dhiman G, Kaur A (2019) STOA: a bio-inspired based optimization algorithm for industrial engineering problems. Eng Appl Artif Intell 82:148-174

30. Satnam K, Awasthi LK, Sangal AL, Dhiman G (2020) Tunicate swarm algorithm: a new bio-inspired based metaheuristic paradigm for global optimization. Eng Appl Artif Intell 90:103541

31. Gaurav D (2019) ESA: a hybrid bio-inspired metaheuristic optimization approach for engineering problems. Eng Comput 1-31

32. Gaurav D, Garg M (2020) MoSSE: a novel hybrid multi-objective meta-heuristic algorithm for engineering design problems. Soft Comput 1-20

33. Gaurav D, Singh KK, Slowik A, Chang V, RizaYildiz A, Kaur A, Garg M (2020) EMoSOA: a new evolutionary multi-objective seagull optimization algorithm for global optimization. Intern $\mathrm{J}$ Mach Learn Cybernet 1-26

34. Gaurav D, Singh KK, Soni M, Nagar A, Dehghani M, Slowik A, Kaur A, Sharma A, Houssein EH, Cengiz K (2020) MOSOA: a new multi-objective seagull optimization algorithm. Exp Syst Appl 114150

35. Dhiman G, Kumar V (2018) Emperor penguin optimizer: a bioinspired algorithm for engineering problems. Knowl-Based Syst 159:20-50

36. Yildirim O, Talo M, Ay B, Baloglu UB, Aydin G, Acharya UR (2019) Automated detection of diabetic subject using pre-trained 2D-CNN models with frequency spectrum images extracted from heart rate signals. Comput Biol Med 113:103387

37. Celik Y, Talo M, Yildirim O, Karabatak M, Acharya UR (2020) Automated invasive ductal carcinoma detection based using deep transfer learning with whole-slide images. Pattern Recogn Lett 133:232-239

38. Chest X-ray images (Pneumonia) https://www.kaggle.com/pault imothymooney/chest-xray-pneumonia 